\title{
Confusion, difficulty concentrating, and slow response induced by ropinirole therapy in an elderly patient with secondary Parkinsonism: a case report
}

\author{
Emilia Sidharta*1 , Ivon Sindu Wijaya ${ }^{1}$, Hanny Cahyadi ${ }^{1,2}$ \\ ${ }^{1}$ Department of Pharmacy National Hospital, Surabaya \\ ${ }^{2}$ Faculty of Pharmacy, University of Surabaya \\ *Corresponding author: sidhartaemilia@gmail.com
}

\begin{abstract}
Background: Ropinirole is a therapy widely used in patients with Parkinson's disease or Restless Leg Syndrome (RLS). A number of research journals report on the long-term use of ropinirole causing side effects to patients. Unfortunately, there are no published studies from Indonesia that document the side effects of ropinirole, especially in elderly subjects.

Case Presentation: This case study reports a number of side effects, such as confusion, difficulty concentrating, and slow response, experienced by a 75-year-old elderly patient who consumed ropinirole 2 $\mathrm{mg}$ as a therapy for secondary Parkinsonism. Prior to using ropinirole, the patient had taken levodopabenserazide, trihexyphenidyl, and pramipexole to overcome Parkinson's for more than 6 years during which no side effects occurred. Side effects appeared after ropinirole therapy was given to the patient, thereby strongly suggesting the administration of ropinirole as the underlying cause of such effects. The assessment methods involved time series data collection followed by causality analysis using the Naranjo Scale, resulting in a score of 6, or Probable. According to some literature, such side effects are probably triggered by the presence of neurotransmitter imbalance, one of which is due to the activation of dopaminergic receptors. Ropinirole is a dopamine agonist drug normally working on dopamine receptors, especially on D2-receptors. An excessive activation of dopaminergic receptors can lead to neuropsychiatric symptoms. In addition, the activation of D2-receptor causes an inhibition of acetylcholine synthesis, resulting in cholinergic deficiency that also becomes one of the triggers for neuropsychiatric symptoms.

Conclusion: The analysis using the Naranjo Scale shows a score of 6, indicating a probable association of confusion, difficulty concentrating, and slow response with ropinirole in this elderly patient with secondary Parkinsonism.
\end{abstract}

Keywords: side effect, secondary Parkinsonism, ropinirole, elderly, case report

\section{Introduction}

Parkinson's is one of the most common neurodegenerative diseases in elderly patients (Willis, 2013). Discovered in 1960, Levodopa has since become the main therapy widely prescribed for Parkinson's patients. However, as the world of knowledge develops, a large number of other drugs begin to be used for Parkinson's as both primary therapy and adjunctive therapy, and one of them is in the dopamine agonist class.

In practice, dopamine agonists play an important role in Parkinson's therapy. Literature suggests that adding dopamine agonists to the regimen can reduce the symptoms of dyskinesia in 
patients (Konta \& Frank, 2008). In general, dopamine agonists are classified into two main groups: dopamine agonists with ergotic structure and dopamine agonists with non-ergotic structure.

Since its approval by the FDA in 1997 and Badan Pengawas Obat dan Makanan (BPOM) in 2012, ropinirole, a non-ergoline dopamine agonist drug, has been widely used as a treatment for Parkinson's and Restless Leg Syndrome (RLS). The effectiveness of ropinirole has been proven through various published studies, but the side effects were seldom reported.

Until this article was written, only two case reports documenting the side effects of ropinirole were found globally (Grover \& Ghosh, 2010; Pal et al., 2012) while there were no reports of such adverse events in Indonesia. Documentation and publication of the side effects of drugs are important to increase pharmacist's or other healthcare professional's awareness of patients with especially long-term use of drugs.

\section{Methodology}

We report on a case of ropinirole side effects and describe the pharmacological background. The assessment methods used were time series data collection followed by causality analysis using the Naranjo Adverse Drug Probability Scale.

\section{Case report}

Mr. U is a 75-year-old man diagnosed with Parkinson's since approximately three years ago. During this period, the patient was treated by Doctor $\mathrm{F}$ and prescribed the following drugs: trihexyphenidyl $2 \mathrm{mg}$ ( $3 \mathrm{x}$ daily 1 tablet), pramipexole $0.125 \mathrm{mg}$ ( $3 \mathrm{x}$ daily 1 tablet), and a combination of levodopa $100 \mathrm{mg}$ - benserazide $25 \mathrm{mg}$ ( $3 \mathrm{x}$ daily 1 tablet). While consuming the medicines, his Parkinson's symptoms were controlled, and he did not experience any side effects. Based on the medical history, the patient also had other diseases, including Benign Prostatic Hyperplasia (BPH) treated with mirabegron, tamsulosin, silodosin, and dutasteride. In addition, he also routinely used other drugs: candesartan 8mg (1x daily 1 tablet), coenzyme 100 (1x daily 1 tablet), bisoprolol 2.5mg (1x daily 1tablet), and clopidogrel $75 \mathrm{mg}$ (1x daily 1 tablet). The only laboratory examination

performed on the patient was serum creatinine carried out on November $22^{\text {nd }}, 2017$ and resulting in normal serum creatinine $(1.1 \mathrm{mg} / \mathrm{dL})$.

In September 2018, the patient came to other doctors (doctor B and doctor S) because he had such symptoms as tremors, limb rigidity, bradykinesia, postural instability, and retropulsion. After a number of examinations, both doctors gave the same diagnosis as the previous one, thereby allowing the patient to continue the treatment from Dr. F without any additional new regimen. In November 2018, the patient returned to Dr. F, and the diagnosis changed from Parkinson's disease 
to secondary Parkinsonism, resulting in addition of ropinirole $2 \mathrm{mg}$ ( $1 \mathrm{x}$ daily 1 tablet) to the ongoing treatment.

In June 2019, the patient's family came to the pharmacy to report the discontinuation of ropinirole use as he developed some symptoms, including confusion, difficulty concentrating, and slow response. The patient then tried to take the medicine every 3 days and once a week, thereby resulting in reduction but persistence of symptoms which led to a stop of ropinirole consumption. Having received such report, the pharmacist then analyzed the possibility of side effects using the Naranjo Adverse Drug Probability Scale, thus obtaining a score of 6 which indicated Probable. The pharmacist then discussed with the doctor who eventually agreed to stop administering ropinirole.

\section{Discussion}

Parkinson's is a long-term degenerative disease of which the causes remain unknown but are believed to involve both genetic and environmental factors. Some literature stated that one of the suspected causes of Parkinson's is the loss of cells containing melanin in the substantia nigra located in the brain, leading to degenerative loss of neurons in the corpus striatum of mesencephalon. This structure also functions as a producer of neurotransmitters, including dopamine. Due to such cell disappearance, dopamine is not sufficiently produced in the brain, resulting in movement disorders that are commonly found in Parkinson's patients (Meder et al., 2019).

In the case reported herein, a 75-year-old male patient had been diagnosed with Parkinson's for more than three years, which then developed into secondary Parkinsonism. Based on literature studies, patients with secondary parkinsonism have the same symptoms as those with Parkinson's disease, but the causes vary from drugs or exposure to hazardous chemicals to other diseases (Falvo \& Holland, 2018). In this case, however, the cause of secondary Parkinsonism in the patient was not explicitly mentioned.

During the treatment for Parkinson's and secondary Parkinsonism, the patient was given a combination of trihexyphenidyl $2 \mathrm{mg}$ ( $3 \mathrm{x}$ daily 1 tablet), pramipexole $0.125 \mathrm{mg}$ ( $3 \mathrm{x}$ daily 1 tab), and levodopa 100mg-benserazide 25mg combination (3x daily 1 tab). The regimen has been used for more than three years during which the patient's condition was controlled and no drug side effects developed. When ropinirole $2 \mathrm{mg}$ was added to the therapeutic regimen, however, the patient started experiencing side effects, such as confusion, difficulty concentrating, and slow response. Literature suggests that the side effects of these drugs may be categorized into neuropsychiatric symptoms. Neuropsychiatric is a term that refers to a variety of medical conditions involving neurology and psychiatry (Sachdev \& Mohan, 2013). The hypothesis of drug side effects was strengthened through an analysis using the Naranjo Adverse Drug Probability Scale, which yielded a 
score of 6 indicating Probable (Table 1). Probable means the reaction follows a reasonable temporal sequence after a drug, follows a recognized response to the suspected drug, is confirmed by withdrawal but not by exposure to the drug, and cannot be reasonably explained by the known characteristics of the patient's clinical state.

References suggest that neuropsychiatric side effects are likely to arise due to various factors. One approach that can be used in this case is through the mechanism of delirium. In this report, the patient experienced some symptoms similar to those found in delirium patients, but they were not necessarily identified as delirium. Further examination and analysis are required to diagnose delirium in patients.

Some of the mechanisms contributing to delirium include cholinergic deficiency, disruption in the synthesis of acetylcholine, or neurotransmitter imbalance (Hshieh et al., 2008). Neurotransmitter imbalance involves acetylcholine, dopamine, or gamma aminobutyric acid (GABA) (Alagiakrishnan \& Wiens, 2004). Some neurotransmitters in the brain such as dopamine, norepinephrine, and serotonin play a role in arousal and sleep-wake cycle as well as in mediating psychological responses to the presence of stimuli. Excessive stimulation of dopaminergic receptors results in decreased acetylcholine, thus leading to a variety of neuropsychiatric symptoms. This phenomenon occurs due to an overlap of the dopaminergic and cholinergic pathways in the brain, thereby suggesting a close relationship between acetylcholine and dopamine activities (Hshieh et al., 2008).

Ropinirole is a group of dopamine agonist drugs that acts primarily on Dopamine D2 receptors. Activation of dopamine receptors decreases acetylcholine synthesis, triggering obstacles in the cholinergic pathway and resulting in neuropsychiatric symptoms found in the patient concerned. In addition, physiological changes in the body also play a role in the existence of neuropsychiatric symptoms. In elderly patients, the response to drugs can be influenced by the physiological changes due to aging. Some factors that can affect include increased body fat, decreased lean body mass and water, decreased albumin, and decreased Glomerular Filtration Rate (GFR) (Alagiakrishnan \& Wiens, 2004).

Several other co-morbidities also affect the action of drugs in the elderly. For example, in patients with liver disorders, albumin synthesis usually decreases, thereby reducing protein binding and increasing the volume of distribution of some drugs. A decrease in kidney function also reduces drug elimination, which eventually increases the concentration of some drugs. Increased blood concentration of drugs can escalate drug effects thus triggering neuropsychiatric symptoms such as those found in delirium patients (Alagiakrishnan \& Wiens, 2004). In this case, such reasons can also 
affect the occurrence of side effects as mentioned in Naranjo Scale point 5. In addition, administration of other drugs and other diseases can contribute to the development of side effects. Therefore, further research is required to investigate the exact cause of the side effects experienced by the patient.

Table 1. Naranjo Adverse Drug Reaction Probability Scale

\begin{tabular}{|c|c|c|c|c|c|}
\hline No. & Question & Yes & No & $\begin{array}{l}\text { Don't } \\
\text { Know }\end{array}$ & Score \\
\hline 1 & $\begin{array}{l}\text { Are there previous conclusive reports on this } \\
\text { reaction? }\end{array}$ & +1 & 0 & 0 & 1 \\
\hline 2 & $\begin{array}{l}\text { Did the adverse event appear after the } \\
\text { suspected drug was administered? }\end{array}$ & +2 & -1 & 0 & 2 \\
\hline 3 & $\begin{array}{l}\text { Did the adverse reaction improve when the } \\
\text { drug was discontinued or a specific antagonist } \\
\text { was administered? }\end{array}$ & +1 & 0 & 0 & 1 \\
\hline 4 & $\begin{array}{l}\text { Did the adverse reaction reappear when the } \\
\text { drug was re-administered? }\end{array}$ & +2 & -1 & 0 & 2 \\
\hline 5 & $\begin{array}{l}\text { Are there alternative causes (other than the } \\
\text { drug) that could solely have caused the } \\
\text { reaction? }\end{array}$ & -1 & +2 & 0 & -1 \\
\hline 6 & $\begin{array}{l}\text { Did the reaction reappear when a placebo was } \\
\text { given? }\end{array}$ & -1 & 0 & 0 & 0 \\
\hline 7 & $\begin{array}{l}\text { Was the drug detected in the blood (or other } \\
\text { fluids) in a concentration known to be toxic? }\end{array}$ & +1 & 0 & 0 & 0 \\
\hline 8 & $\begin{array}{l}\text { Was the reaction more severe when the dose } \\
\text { was increased, or less severe when the dose } \\
\text { was decreased? }\end{array}$ & +1 & 0 & 0 & 1 \\
\hline 9 & $\begin{array}{l}\text { Did the patient have a similar reaction to the } \\
\text { same or similar drugs in any previous } \\
\text { exposure? }\end{array}$ & +1 & 0 & 0 & 0 \\
\hline 10 & $\begin{array}{l}\text { Was the adverse event confirmed by objective } \\
\text { evidence? }\end{array}$ & +1 & 0 & 0 & 0 \\
\hline
\end{tabular}

Interpretation of Scores:

Total score $\leq 0$ : doubtful, total score 1-4: possible, total score 5-8: probable, total score $>9$ : definite

The influence of neurotransmitter imbalance on neuropsychiatric symptoms can be strengthened by the presence of Serum Anticholinergic Activity (SAA) levels. However, in this study, laboratory tests have not been conducted to determine the quantitative levels of the neurotransmitters in the body. Therefore, it is recommended to perform a more in-depth examination related to SAA level to confirm the existence of these side effects due to the use of ropinirole. 


\section{Conclusion}

Ropinirole causes various neuropsychiatric side effects, such as confusion, difficulty concentrating, and slow response. The monitoring of drug therapy needs to be performed by health professionals with regard to the use of Parkinson's drugs, especially in elderly patients. Changes in the physiological profile, pharmacokinetics, and pharmacodynamics of the body will influence the effects of drugs in the body. In addition, adjustment of individual doses according to each patient's characteristics is extremely essential.

\section{References}

Alagiakrishnan, K., \& Wiens, C. A. (2004). An approach to drug induced delirium in the elderly. Postgraduate medical journal, 80(945), 388-393. doi:10.1136/pgmj.2003.017236

Falvo, D. R., \& Holland, B. E. (2018). Medical and psychosocial aspects of chronic illness and disability. Burlington: Jones and Bartlett Publishers.

Grover, S., \& Ghosh, A. (2010). A case of ropinirole-induced psychosis. Primary care companion to the Journal of clinical psychiatry, 12(6), PCC.10100987. doi:10.4088/PCC.10100987gre

Hshieh, T. T., Fong, T. G., Marcantonio, E. R., \& Inouye, S. K. (2008). Cholinergic deficiency hypothesis in delirium: a synthesis of current evidence. The journals of gerontology. Series A, Biological sciences and medical sciences, 63(7), 764-772. doi:10.1093/gerona/63.7.764

Konta, B., \& Frank, W. (2008). The treatment of Parkinson's disease with dopamine agonists. GMS health technology assessment, 4, Doc05-Doc05. Retrieved from https://pubmed.ncbi.nlm.nih.gov/21289911

Meder, D., Herz, D. M., Rowe, J., Lehericy, S., \& Siebner, H. R. (2019). The role of dopamine inthe brain-lesson learned from Parkinson's disease. NeuroImage, 190. doi:https://doi.org/10.1016/j.neuroimage.2018.11.021

Pal, K., Smith, A., Hayes, J., \& Chakraborty, A. (2012). Othello syndrome secondary to ropinirole: a case study. Case reports in psychiatry, 2012, 353021-353021. doi:10.1155/2012/353021

Sachdev, P. S., \& Mohan, A. (2013). Neuropsychiatry: where are we and where do we go from here? Mens sana monographs, 11(1), 4-15. doi:10.4103/0973-1229.109282

Willis, A. W. (2013). Parkinson disease in the elderly adult. Missouri medicine, 110(5), 406-410. 